\title{
Pathway-specific dopaminergic deficits in a mouse model of Angelman syndrome
}

\author{
Thorfinn T. Riday, ${ }^{1,2}$ Elyse C. Dankoski, ${ }^{1}$ Michael C. Krouse, ${ }^{3}$ Eric W. Fish, ${ }^{3}$ Paul L. Walsh, ${ }^{4}$ \\ Ji Eun Han, ${ }^{2}$ Clyde W. Hodge, ${ }^{1,5}$ R. Mark Wightman, ${ }^{1,4,6}$ \\ Benjamin D. Philpot, ${ }^{1,2,6,7}$ and C.J. Malanga ${ }^{1,3,5,7}$
}

\begin{abstract}
${ }^{1}$ Curriculum in Neurobiology, University of North Carolina at Chapel Hill, Chapel Hill, North Carolina, USA. ${ }^{2}$ Department of Cell Biology and Physiology and ${ }^{3}$ Department of Neurology, University of North Carolina School of Medicine, University of North Carolina, Chapel Hill, North Carolina, USA. ${ }^{4}$ Department of Chemistry, ${ }^{5}$ Bowles Center for Alcohol Studies, 6 UNC Neuroscience Center, and 7 UNC Carolina Institute for Developmental Disabilities, University of North Carolina at Chapel Hill, Chapel Hill, North Carolina, USA.
\end{abstract}

\begin{abstract}
Angelman syndrome (AS) is a neurodevelopmental disorder caused by maternal deletions or mutations of the ubiquitin ligase E3A (UBE3A) allele and characterized by minimal verbal communication, seizures, and disorders of voluntary movement. Previous studies have suggested that abnormal dopamine neurotransmission may underlie some of these deficits, but no effective treatment currently exists for the core features of AS. A clinical trial of levodopa (L-DOPA) in AS is ongoing, although the underlying rationale for this treatment strategy has not yet been thoroughly examined in preclinical models. We found that AS model mice lacking

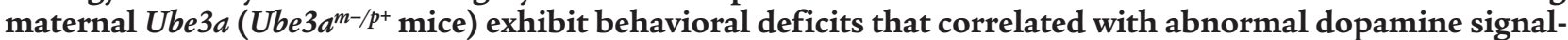
ing. These deficits were not due to loss of dopaminergic neurons or impaired dopamine synthesis. Unexpectedly, Ube $3 a^{m-/ p^{+}}$mice exhibited increased dopamine release in the mesolimbic pathway while also exhibiting a decrease in dopamine release in the nigrostriatal pathway, as measured with fast-scan cyclic voltammetry. These findings demonstrate the complex effects of UBE3A loss on dopamine signaling in subcortical motor pathways that may inform ongoing clinical trials of L-DOPA therapy in patients with AS.
\end{abstract}

\section{Introduction}

Angelman syndrome (AS) is a neurodevelopmental disorder characterized by intellectual disability, profound language impairment, seizures, and a propensity for a happy disposition (1-3). AS results from loss of function of the maternally inherited $U B E 3 A$ allele at the 15q11-q13 locus (4-9). The UBE3A gene encodes a HECT domain E3 ubiquitin ligase (UBE3A, also known as E6AP) involved in protein degradation through the ubiquitin-proteasome pathway $(4,10)$. Clinical treatment of AS commonly includes pharmacotherapy for seizures, problem behaviors, and motor dysfunction (11). Although treatments for AS are limited, a case study of 2 adults with AS found that levodopa (L-DOPA) administration dramatically improved resting tremor and rigidity (12), leading to a clinical trial of L-DOPA in individuals with AS (13).

There are few published studies validating the rationale for using L-DOPA to treat parkinsonian features in AS. AS model mice lacking maternal Ube3a (Ube3 $a^{m-p^{+}}$mice) were reported to have reduced dopamine cell number in the substantia nigra pars compacta (SNc) by 7 to 8 months of age (14). In Drosophila, UBE3A has been shown to regulate GTP cyclohydrolase I, an essential enzyme in dopamine biosynthesis (15). However, little is known about the function of mesolimbic or nigrostriatal dopamine pathways in AS, which have vital roles in several of the behaviors or motor symptoms commonly managed with pharmacotherapy, including hyperactivity, impulsivity, tremor, and rigidity. A survey of psychoactive drugs used in patients with AS reported that the majority responded poorly to stimulant medications (16), most of which act by increasing available extracellular dopamine levels.

Conflict of interest: The authors have declared that no conflict of interest exists. Citation for this article: J Clin Invest. 2012;122(12):4544-4554. doi:10.1172/JCI61888.
We examined dopamine-dependent behaviors as well as dopamine synthesis, content, and release in the mesolimbic and nigrostriatal pathways of AS model mice. Ube $3 a^{m-/ p^{+}}$mice were more sensitive to brain stimulation reward (BSR) but less sensitive to the effects of drugs that increase extracellular dopamine in behavioral measures of both reward and locomotion. Surprisingly, we found increased dopamine release in the mesolimbic system but decreased release in the nigrostriatal system. These changes in dopaminergic function were not accounted for by differences in dopaminergic cell number or differences in tyrosine hydroxylase levels or dopamine content in the terminal fields of the nucleus accumbens (NAc) or dorsal striatum. Our findings raise the possibility that similar effects on dopaminergic systems may occur in humans and may inform ongoing and future clinical trials of L-DOPA in individuals with AS.

\section{Results}

Ube $3 a^{m-/ p^{+}}$mice are more sensitive to rewarding electrical brain stimulation. Activity of mesolimbic dopaminergic neurons in the midbrain ventral tegmental area (VTA) is critical for the perception of reward $(17,18)$. To determine whether loss of UBE3A alters mesolimbic dopamine function, Ube $3 a^{m-/ p^{+}}$and WT mice were implanted with stimulating electrodes in the medial forebrain bundle (MFB) and trained to perform operant intracranial selfstimulation (ICSS) by turning a wheel (Supplemental Figure 1A; supplemental material available online with this article; doi:10.1172/JCI61888DS1). Thresholds for perception of BSR were determined before and after administration of drugs that increase extracellular dopamine levels (Figure 1A). Ube $3 a^{m-/ p^{+}}$ mice showed a leftward shift of the baseline charge-response curve (Figure 1B), indicating that these mice required less charge than WT littermates to sustain the same degree of wheel turn- 

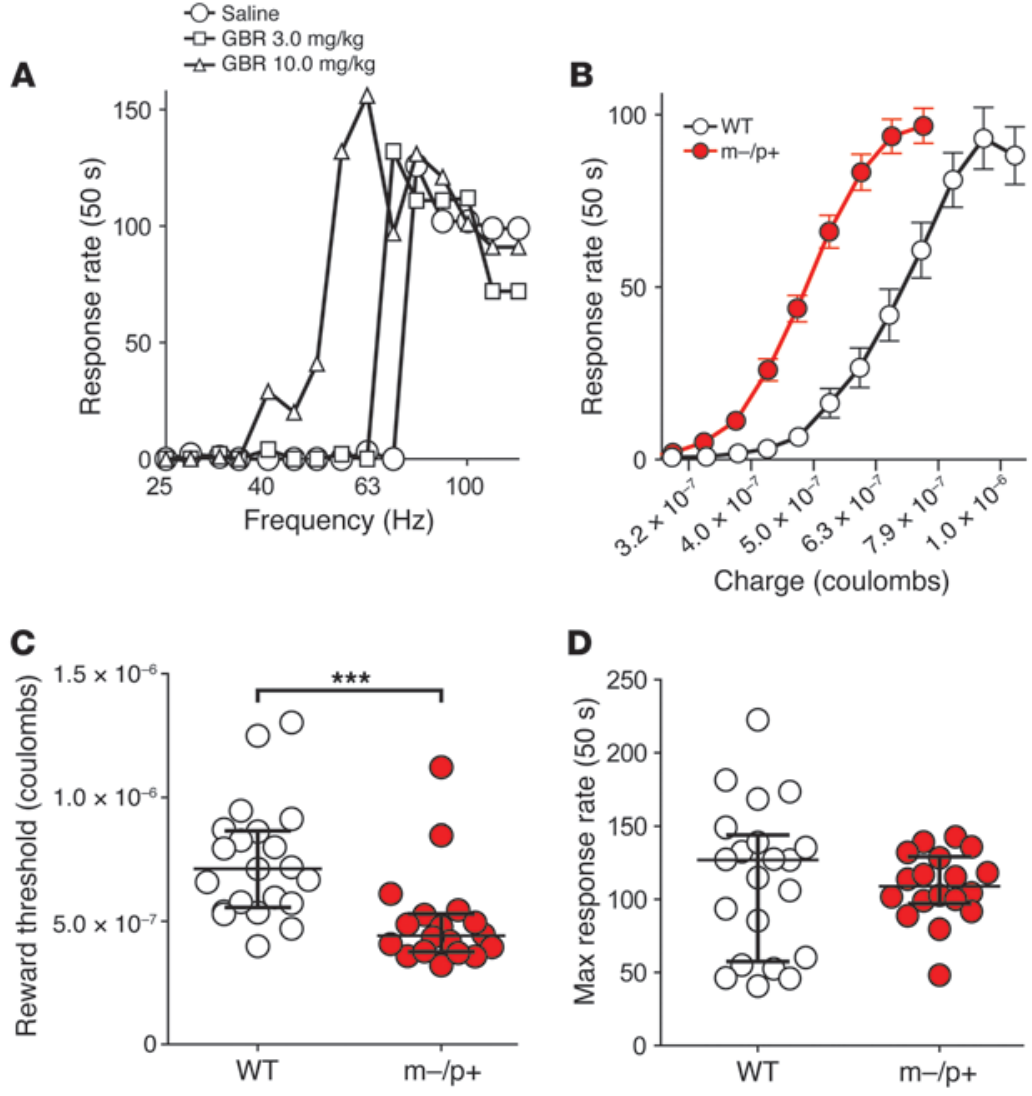

E

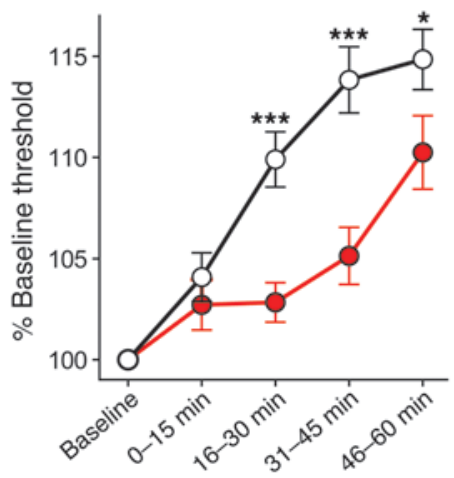

D
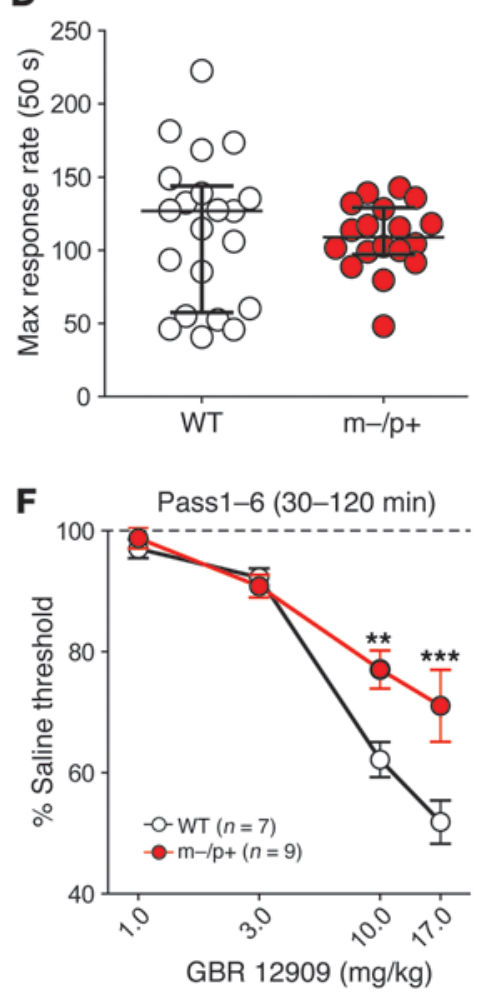

\section{Figure 1}

Ube $3 a^{m-/ p+}$ mice are more sensitive to BSR but less sensitive to dopaminergic potentiation of BSR. (A) Representative ICSS rate-frequency curves in a WT mouse. Injection (i.p.) of the DAT antagonist GBR 12909 dose-dependently increases responding for rewarding electrical current at lower stimulus frequencies. (B) Rate-frequency curves expressed as charge (Q) delivery at each frequency $(\mathrm{Hz})$ from $U b e 3 a^{m-/ p+}$ mice are shifted to the left compared with those of WT littermates. (C) Ube3a $a^{m-1 p+}$ mice require significantly less $\left({ }^{* * *} P<0.001\right)$ charge to evoke the same degree of responding as WT mice at reward threshold frequencies (EF50). (D) The maximum rate of operant responding for rewarding brain stimulation is comparable between genotypes $(P>0.05)$. (E) Ube3am-/p+ mice maintain a lower reward threshold over time (16-30 minutes, ${ }^{* \star} P<0.001 ; 31-45$ minutes, ${ }^{\star * *} P<0.001 ; 46-60$ minutes, $\left.{ }^{*} P=0.026\right)$. (F) WT mice exhibit greater potentiation of rewarding brain stimulation expressed as lower reward thresholds than Ube3a ${ }^{m-1 p+}$ mice following $10.0 \mathrm{mg} / \mathrm{kg}\left({ }^{* *} P=0.002\right)$ and $17.0 \mathrm{mg} / \mathrm{kg}\left({ }^{* * *} P<0.001\right)$ GBR 12909 (i.p.). Error bars indicate \pm SEM in $\mathbf{B}, \mathbf{E}$, and $\mathbf{F}$ and the median and interquartile ranges in $\mathbf{C}$ and $\mathbf{D}$. ing (Figure 1C; $U=59.0, P<0.001$ ). There was no difference in the maximum rate of operant responding between genotypes (Figure 1D), demonstrating that voluntary motor function required for ICSS was unimpaired in Ube $3 a^{m-/ p^{+}}$mice. Ube $3 a^{m-/ p^{+}}$ mice also sustained a lower reward threshold for longer than WT littermates (16-30 minutes, $P<0.001$; 31-45 minutes, $P<0.001$; 46-60 minutes, $P=0.026$; Figure $1 \mathrm{E})$.

Ube $3 a^{m-/ p^{+}}$mice are less sensitive to dopaminergic manipulation of BSR. Drugs that enhance extracellular dopamine availability increase the potency of BSR, measured as a lowered BSR threshold (Supplemental Figure 1, B and C). To determine whether the increase in reward sensitivity in $U b e 3 a^{m-/ p^{+}}$mice was due to changes in dopamine neurotransmission, we investigated the effects of pharmacological manipulation on BSR threshold. The nonselective monoamine reuptake blocker, cocaine, similarly lowered BSR thresholds in both genotypes at the peak of its effect from 0 to 15 minutes after i.p. administration (Figure 2, A and B, and Supplemental Figure $2 \mathrm{~A}$ ), but the reward-potentiating effects of cocaine decayed more slowly in Ube $3 a^{m-/ p^{+}}$mice (Figure 2C). Maximum operant response rates showed a greater increase following cocaine administration in WT mice at $10.0 \mathrm{mg} / \mathrm{kg}$ cocaine (31-45 minutes, $P=0.028)$ and $17.0 \mathrm{mg} / \mathrm{kg}$ cocaine (31-45 minutes, $P=0.001$; Supplemental Figure $3 \mathrm{~A}$ ), indicating that cocaine effects on operant motor behavior are reduced in Ube $3 a^{m-/ p^{+}}$mice. The highly selective dopamine transporter (DAT) blocker GBR 12909 reduced reward threshold similarly to cocaine but remained active for over 2 hours following administration. GBR 12909 lowered reward threshold significantly less in $U b e 3 a^{m-/ p^{+}}$mice, revealing a more pronounced difference in potentiation of BSR than that seen with cocaine $(10.0 \mathrm{mg} / \mathrm{kg}, P=0.002 ; 17.0 \mathrm{mg} / \mathrm{kg}, P<0.001 ;$ Figure $1 \mathrm{~F}$ and $\mathrm{Sup}-$ 

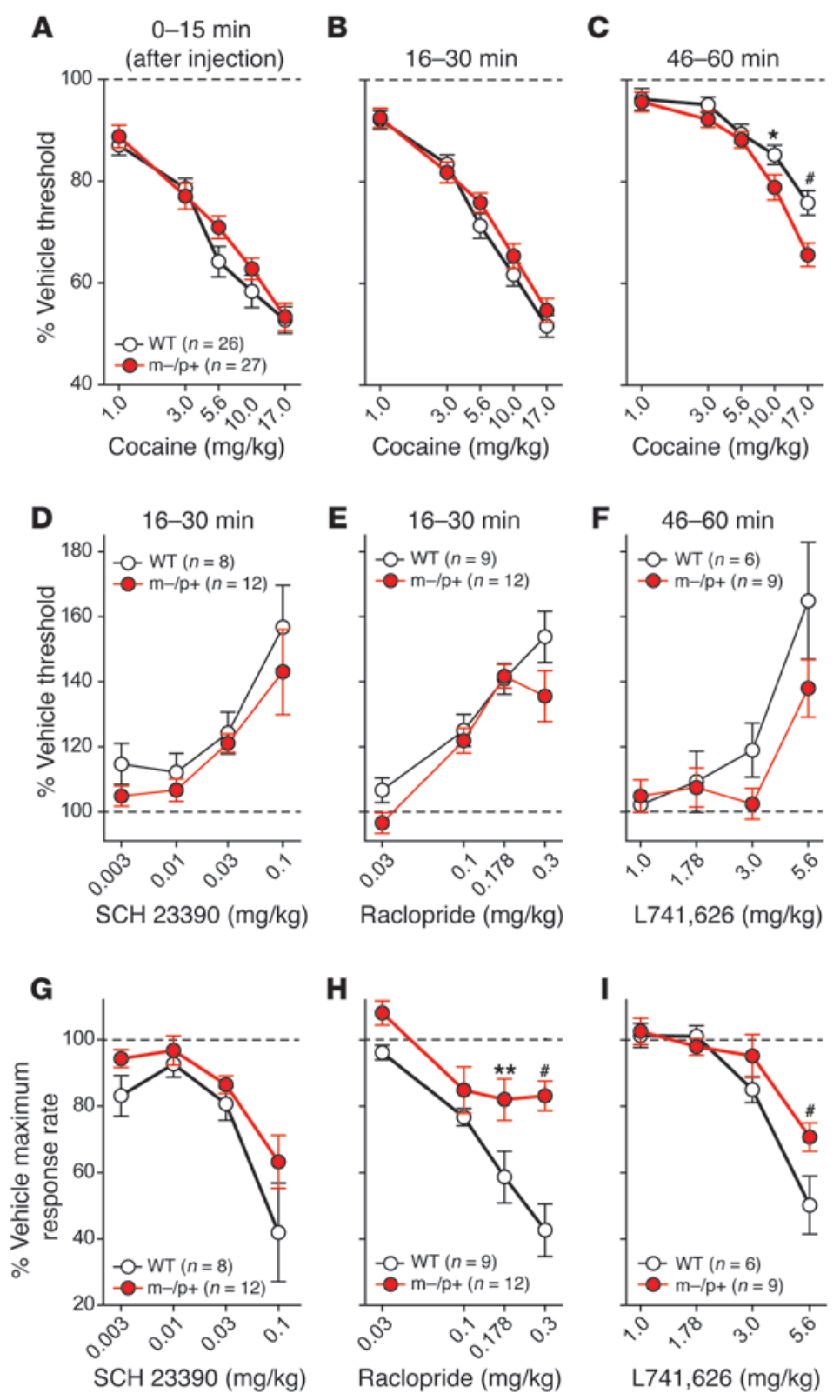

\section{Figure 2}

Ube3a $a^{m-/ p+}$ mice exhibit normal BSR threshold responses to cocaine and selective D1 and D2 dopamine receptor antagonists but decreased sensitivity to D2-dependent motor impairment. BSR threshold was determined following i.p. administration of $(\mathbf{A}-\mathbf{C})$ cocaine, (D) the D1 receptor antagonist $\mathrm{SCH} 23390$, or the D2-selective antagonists (E) raclopride or (F) L741,626. (A-C) Cocaine had similar potency on BSR threshold in both genotypes at its peak effect (0-15 minutes), but its rewarding effects decayed more slowly in Ube3a ${ }^{m-1 p+}$ mice (46-60 minutes, $10.0 \mathrm{mg} / \mathrm{kg},{ }^{*} P=0.025$; $\left.17.0 \mathrm{mg} / \mathrm{kg}, "{ }^{\prime} P<0.001\right)$. (D) The D1 antagonist SCH 23390, (E) the D2-like antagonist raclopride, or (F) the highly D2-selective antagonist $L 741,626$ equally elevated reward thresholds of WT and mutant mice. (G) However, while D1 receptor antagonism had similar depressant effects on maximum operant response rates of both genotypes, antagonism of D2 receptors with either $(\mathbf{H})$ raclopride $(0.178 \mathrm{mg} / \mathrm{kg}$, $\left.{ }^{\star *} P=0.005 ; 0.3 \mathrm{mg} / \mathrm{kg},{ }^{\#} P<0.001\right)$ or (I) L741,626 (5.6 mg/ $\left.\mathrm{kg},{ }^{\#} P<0.001\right)$ had greater depressant effects on maximum response rates of WT mice. Error bars indicate \pm SEM.

tor antagonist L741,626 (Figure 2F) were also similar between genotypes (see also Supplemental Figure 2, D and $\mathrm{E})$. However, the depressant effect of both raclopride (16-30 minutes, $0.178 \mathrm{mg} / \mathrm{kg}, P=0.005 ; 0.3 \mathrm{mg} / \mathrm{kg}$, $P<0.001$; Figure $2 \mathrm{H}$ and Supplemental Figure 3D) and L741,626 (46-60 minutes, 5.6 mg/kg, $P<0.001$; Figure $2 \mathrm{I}$ and Supplemental Figure $3 \mathrm{E}$ ) on maximum operant response rate was blunted in $U b e 3 a^{m-/ p^{+}}$mice.

Ube $3 a^{m-/ p^{+}}$mice are less sensitive to cocaine-stimulated locomotor activity. To further assess dopamine-related behavior in Ube $3 a^{m-/ p^{+}}$mice, we performed locomotor sensitization experiments using cocaine $(5.6,10.0$, or $17.0 \mathrm{mg} /$ kg i.p.) as a tool to evaluate both acute locomotor stimulation and adaptation to repeated drug exposure (Figure 3). Cocaine has a rapid onset of action, with brain concentrations peaking approximately 5 minutes after injection, and a half-life of approximately 15 minutes (19). Therefore, we used the total activity in the first 15 minutes following injection to compare cocaine-stimulated locomotor activity between genotypes. Total locomotion over the 15 minutes following saline injection was lower in Ube3 $a^{m-/ p^{+}}$mice $(531 \pm 51 \mathrm{~cm})$ than in WT mice $(916 \pm 79 \mathrm{~cm} ; U=266, P<0.001)$, consistent with

plemental Figure 2B). The ability of GBR 12909 to increase the maximum operant response rate was also reduced in $U b e 3 a^{m-/ p^{+}}$ mice at $10.0 \mathrm{mg} / \mathrm{kg}$ (76-90 minutes, $P=0.032 ; 91-105$ minutes, $P=0.018)$ and $17.0 \mathrm{mg} / \mathrm{kg}(76-90$ minutes, $P=0.015 ; 91-105$ minutes, $P=0.004$; Supplemental Figure 3B).

To assess possible differences in dopamine receptor sensitivity in the NAc and other forebrain targets, we measured the potency of selective dopamine receptor antagonists in reducing BSR. We found that the D1 receptor antagonist SCH 23390 elevated BSR thresholds similarly in both genotypes (Figure 2D and Supplemental Figure 2C). The motor depressant effect of SCH 23390 on maximum operant response rate was also similar between WT and Ube $3 a^{m-/ p^{+}}$mice (Figure $2 \mathrm{G}$ and Supplemental Figure 3C), suggesting that dopamine acting through $\mathrm{D} 1$ receptors was unaffected by loss of UBE3A. The reward threshold-elevating effects of the D2/3 receptor antagonist raclopride (Figure $2 \mathrm{E}$ ) and D2-selective recep- decreased motor activity previously described in $U b e 3 a^{m-/ p^{+}}$mice (20). Although a low cocaine dose $(5.6 \mathrm{mg} / \mathrm{kg}$; Figure $3 \mathrm{~A})$ was sufficient in both genotypes to induce locomotor sensitization, defined as greater distance traveled on the challenge/last day compared with that on the first day of administration $(P<0.001)$, we found Ube $3 a^{m-/ p^{+}}$mice to be less sensitized than WT mice (day 4, $P=0.02$; day $5, P=0.031$; challenge, $P=0.031$; Figure $3 \mathrm{D})$. An intermediate cocaine dose $(10.0 \mathrm{mg} / \mathrm{kg}$; Figure $3 \mathrm{~B})$ increased locomotion more in WT mice than in Ube $3 a^{m-p^{+}}$mice for 5 consecutive days after the first day of exposure (day 2, $P=0.009$; day $3, P=0.012$; days $4-6, P<0.001$ ), although this difference was no longer significant on cocaine challenge after 7 days (Figure $3 \mathrm{E}$ ). The largest cocaine dose tested (17.0 mg/kg; Figure 3, C and F) stimulated comparable locomotion on the second day of exposure in both genotypes, suggesting that the reduced cocaine effect in $U b e 3 a^{m-/ p^{+}}$mice was not due to a reduction in maximum cocaine potency or a ceiling effect. 


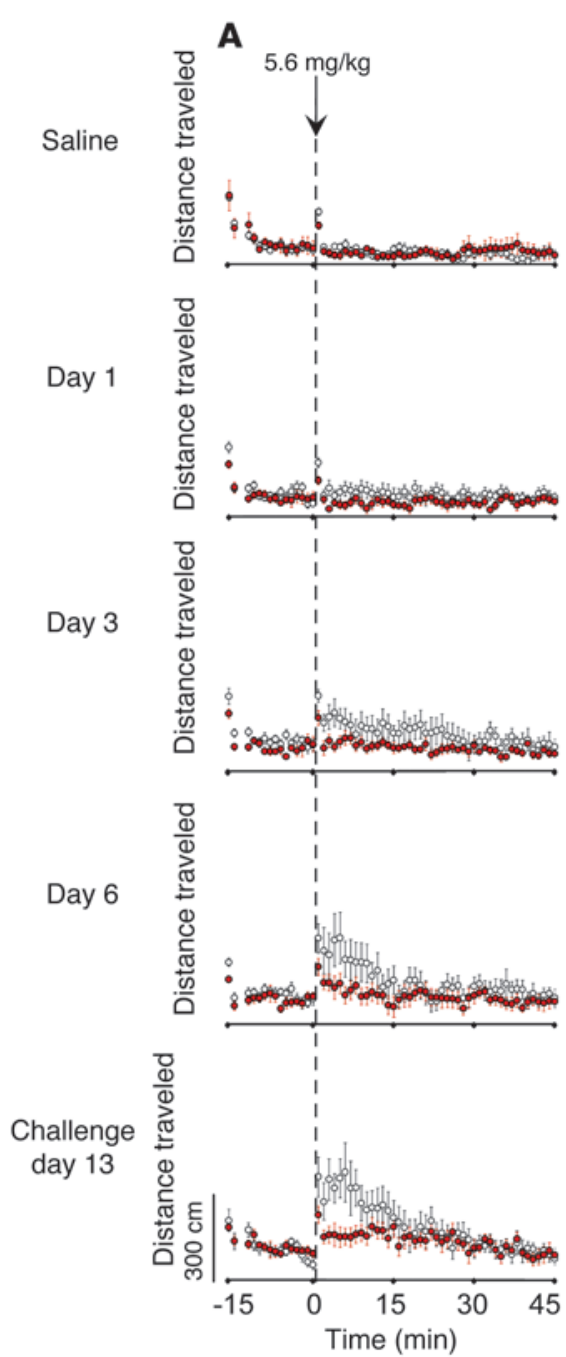

B

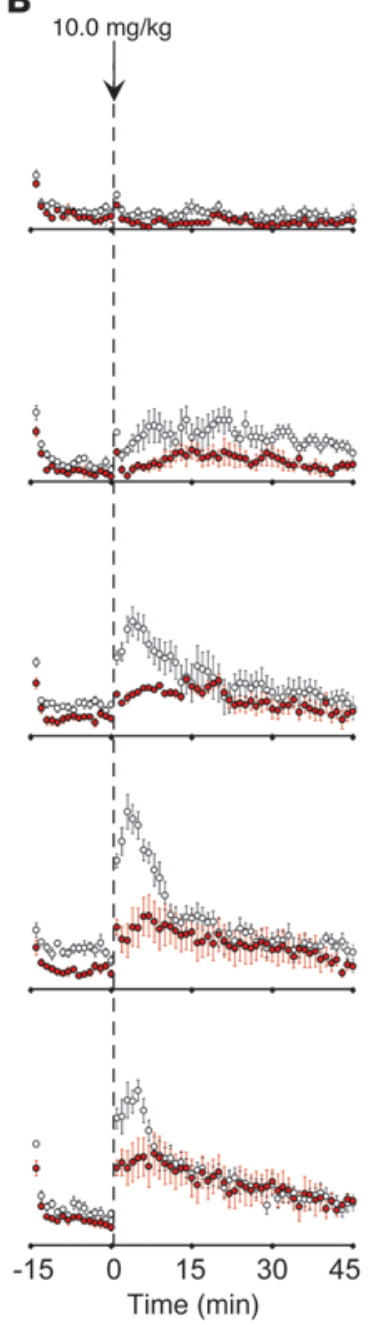

C

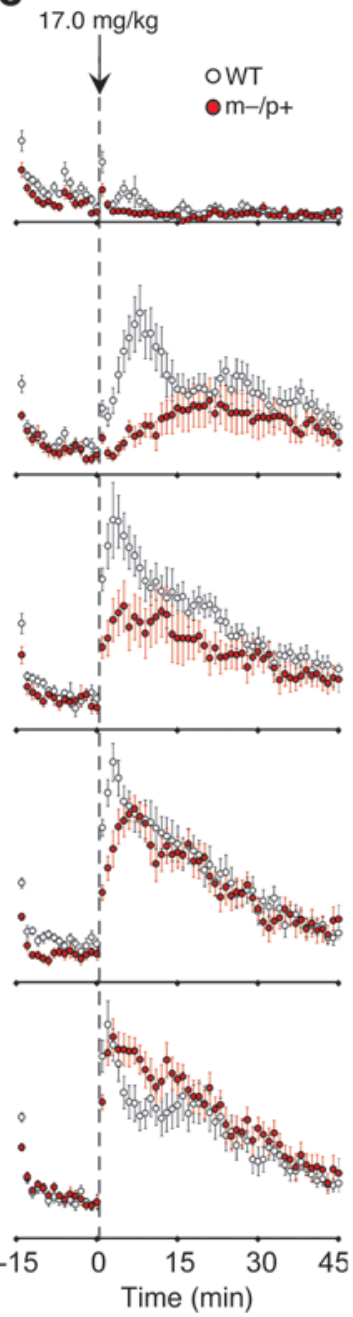

D

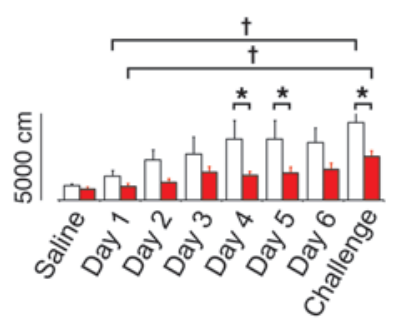

E

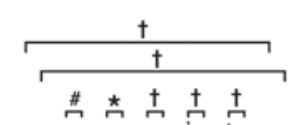

$\mathbf{F}$
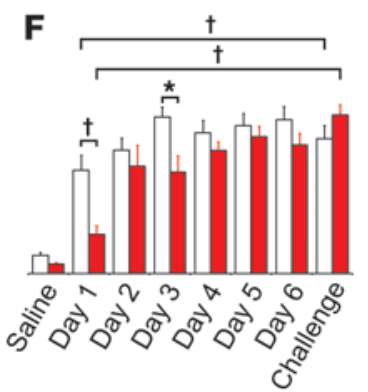

\section{Figure 3}

Cocaine-stimulated locomotion is lower in Ube3a $a^{m-1 p+}$ mice. Psychostimulant-induced locomotion in 3 separate groups of mice ( 12 WT and 12 Ube $3 a^{m-1 p+}$ mice per group) injected with $5.6,10.0$ or $17.0 \mathrm{mg} / \mathrm{kg}$ i.p. cocaine for 6 consecutive days and a final challenge dose 1 week later. (A-C) Distance traveled is binned in 1-minute increments. (D-F) The sum of the first 15 minutes following injection is plotted below each of their respective treatment groups. (D) A deficit in the locomotive response of $U b e 3 a^{m-/ p+}$ mice emerges after 3 days of $5.6 \mathrm{mg} / \mathrm{kg}$ cocaine administration $(P=0.02$; day $5, P=0.031$; challenge, $P=0.031$ ) and $(E)$ by the second day of $10.0 \mathrm{mg} /$ kg cocaine $(P=0.009$; day 3 , $P=0.012$; day 4-6, $P<0.001)$. (F) High-dose $(17.0 \mathrm{mg} / \mathrm{kg})$ cocaine elicits an attenuated response after the first administration in Ube $3 a^{m-1 p+}$ mice that is gone by the fourth day. (D-F) Cocaine-induced sensitization occurred at all 3 doses for both genotypes (day 1 vs. challenge, $P<0.001$ ). Error bars indicate SEM. ${ }^{*} P<0.05$; ${ }^{\#} P<0.01 ;{ }^{\dagger} P<0.001$.
Neuroanatomical and biochemical markers of dopamine are unaltered in Ube $3 a^{m-/ p^{+}}$mice. Projection neurons within the VTA and SNc express both UBE3A and tyrosine hydroxylase (TH), the rate-limiting enzyme in dopamine biosynthesis. We performed immunohistochemistry to confirm maternal imprinting of Ube $3 a$ in the VTA and SNc (Figure 4A) and then quantified TH-positive neurons with design-based stereology in the VTA and SNc. We found no differences in the estimated dopamine cell number in either region at P100 (Table 1 and Figure 4B). We performed Western blots for TH in the NAc and dorsal striatum as a measure of biosynthetic capacity in dopaminergic terminal fields originating from neurons in the VTA or SNc, respectively. TH protein levels were similar between Ube $3 a^{m-/ p^{+}}$and WT mice in the NAc and striatum (Table 2 and Figure 4C). HPLC on tissue homogenates showed no difference in total dopamine content in $U b e 3 a^{m-/ p^{+}}$mice in the NAc or dorsal striatum (Table 2 and Figure 4D). Furthermore, tissue concentrations of DOPAC, the primary acid metabolite of dopamine (Figure 4E), and dopamine/DOPAC concentration ratios (Figure 4F) were comparable between genotypes.

Dopamine transmission is enhanced in the NAc and reduced in the dorsal striatum of Ube $3 a^{m-/ p^{+}}$mice. We performed fast-scan cyclic voltammetry (FSCV) in the NAc and dorsal striatum to determine whether the loss of UBE3A affected phasic dopamine transmission in these limbic and motor terminal regions, respectively 
A TH low magnification
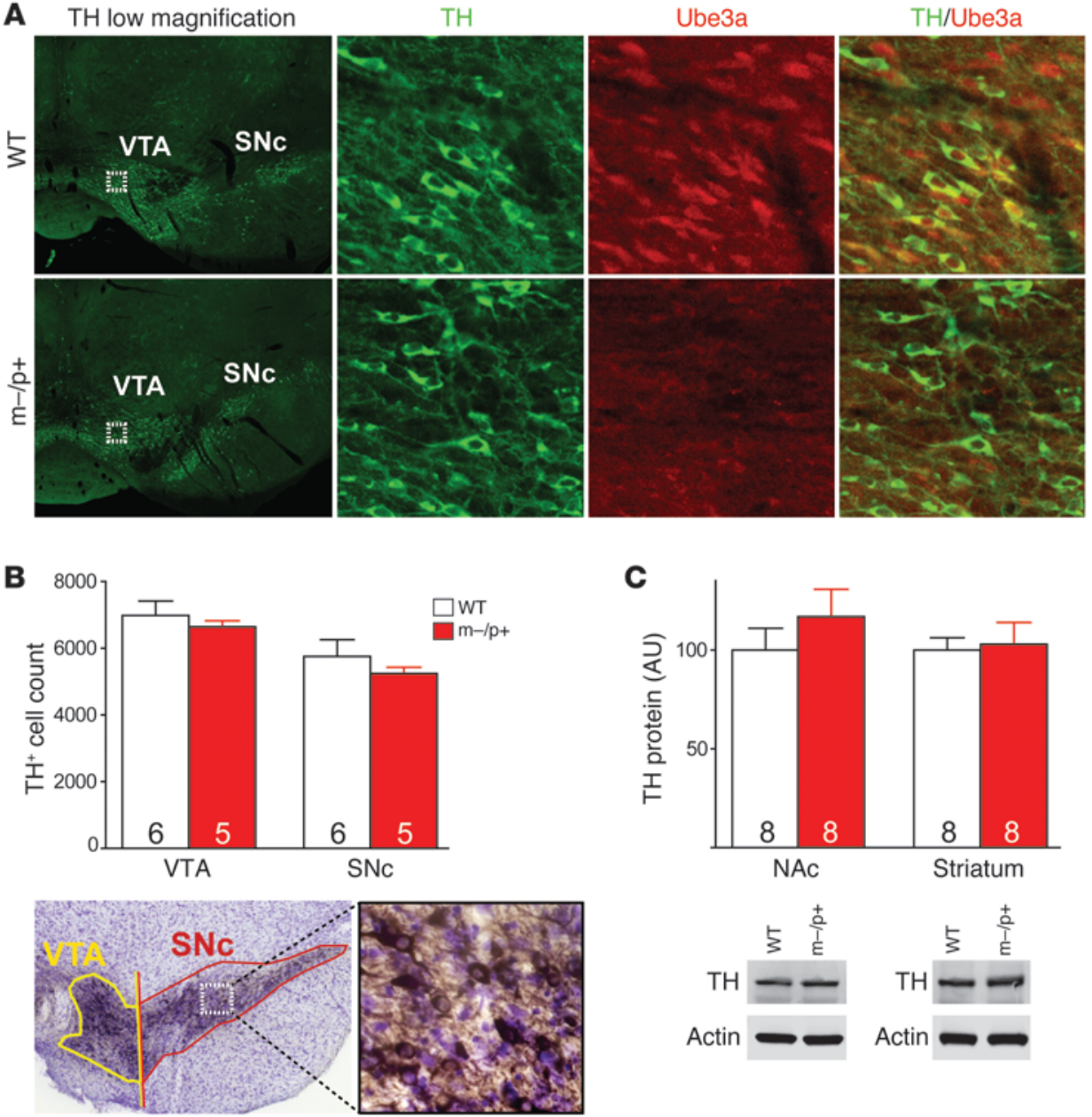

C
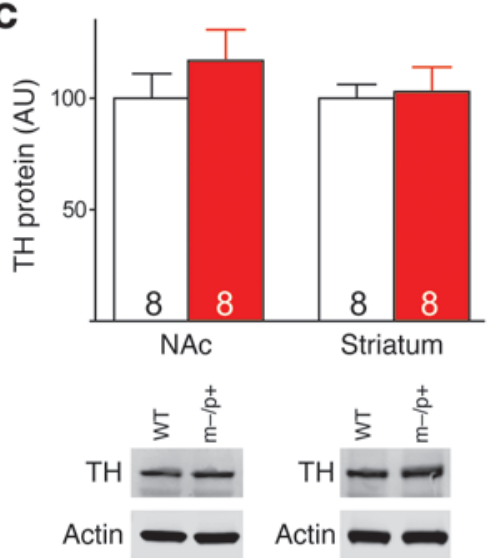

\section{Figure 4}

Biochemical and anatomical markers of the mesolimbic and nigrostriatal dopaminergic pathways are normal in Ube3a ${ }^{m-/ p+}$ mice. (A) Immunohistochemistry showing imprinting of UBE3A in the mesencephalic VTA and SNc and colocalization with TH in WT mice (original magnification, $\times 4$ ). Boxed regions are shown at higher magnification in the second, third, and fourth columns (original magnification, $\times 40$ ). (B) Numbers of TH-positive cells in the VTA and SNc estimated with design-based stereology show no differences between WT and Ube $3 a^{m-/ p+}$ mice. Ventral midbrain image is magnified (original magnification, $\times 4$ ) and the boxed region is shown at higher magnification to the right (original magnification, $\times 40$ ). (C) Quantification of $\mathrm{TH}$, the rate-limiting enzyme in dopamine biosynthesis, from NAc and striatal tissue punches by immunoblotting shows similar levels between the 2 genotypes. (D) HPLC analysis of concentrations of dopamine and $(E)$ its primary acid metabolite DOPAC in NAc and striatal punches from WT and Ube $3 a^{m-/ p+}$ mice shows similar tissue content of dopamine and DOPAC. (F) Dopamine/DOPAC ratios are also equivalent between genotypes in NAc and striatum. Error bars indicate SEM. Numbers in bars represent numbers of mice.
D

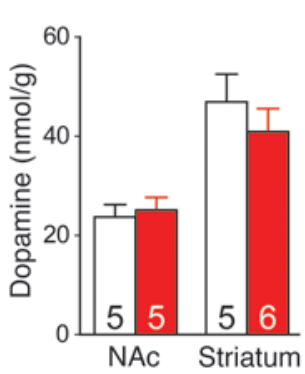

E

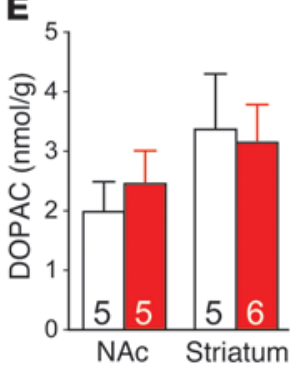

F

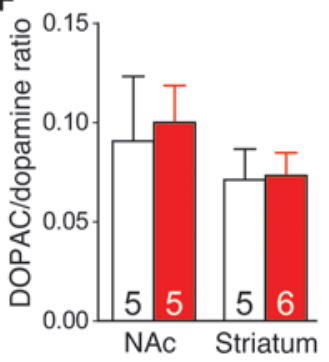

(Figure 5). Stimulation of the MFB resulted in greater dopamine release in the NAc of Ube $3 a^{m-/ p^{+}}$mice across all stimulus frequencies (Figure 5A and Supplemental Figure 4A), with higher maximum extracellular dopamine concentrations obtained at 30 to $60 \mathrm{~Hz}(30 \mathrm{~Hz}, P=0.05 ; 40 \mathrm{~Hz}, P=0.008 ; 50 \mathrm{~Hz}, P=0.045 ; 60$ $\mathrm{Hz}, P=0.004$; Figure 5, C, E, and G). In contrast to that in the $\mathrm{NAc}$, we found reduced dopamine release across all stimulus frequencies (Figure 5B and Supplemental Figure 4B), with lower maximum extracellular dopamine concentration in the dorsal striatum of Ube $3 a^{m-/ p^{+}}$mice at 30 to $50 \mathrm{~Hz}(30 \mathrm{~Hz}, P=0.006 ; 40$ $\mathrm{Hz}, P=0.002$; $50 \mathrm{~Hz}, P=0.015$; Figure 5, D, F, and H). Modeling of the FSCV data did not reveal any differences in dopamine uptake following stimulation in either the NAc or dorsal striatum (data not shown). Administration of the DAT blocker GBR 12909 prior to stimulation caused similar changes to the time course of extracellular dopamine concentrations in the NAc and dorsal striatum (Figure 5, I and J), suggesting no differences in dopamine reuptake between genotypes.

\section{Discussion}

The principal findings of this study are that $U b e 3 a^{m-/ p^{+}}$mice, a model of AS, exhibit increased mesolimbic dopamine release but decreased nigrostriatal dopamine release and decreased behavioral sensitivity to drugs that enhance extracellular dopamine availability. Release of accumbal dopamine is critical to the rewarding effects associated with both BSR and drugs of abuse as well as natural incentives such as food and sex (21). We used ICSS as an experimental model to directly assess motivation and reward and 
Table 1

Anatomical markers of dopamine

\begin{tabular}{ccccc} 
& \multicolumn{2}{c}{ VTA } & \multicolumn{2}{c}{ Substantia nigra } \\
& WT & ${\text { Ube } 3 a^{m-/ p+}}$ & WT & Ube $3 a^{m-/ p+}$ \\
TH $^{+}$cell no. & $6,986 \pm 427.9$ & $6,645 \pm 174.9$ & $5,754 \pm 500.2$ & $5,245 \pm 182.2$ \\
& $(n=6)$ & $(n=5)$ & $(n=6)$ & $(n=5)$ \\
\hline
\end{tabular}

No significant genotype differences were found (mean \pm SEM). tions in patients with autosomal recessive familial Parkinson's disease. Mice lacking either PTEN-induced kinase 1 (Pink1, also known as Park6; ref. 28) or the novel glyoxylase DJ-1 (Park7; refs. 29, 30) exhibit decreased electrically evoked striatal dopamine release in the absence of changes in dopaminergic cell number or dopamine synthesis and metabolism. Decreased spontaneous locomotion is observed with both mutations but is not evident in PINK1-deficient mice until late adulthood (31). Conversely, mice deficient in the E3 ubiquitin to minimize effects from potential sensory processing abnormalities $(18,22)$. We found that $U b e 3 a^{m-/ p^{+}}$mice required significantly less electrical stimulation to attain the same level of responding for BSR as in controls and were able to maintain a higher degree of responding for BSR over time, consistent with the increased dopamine release in the NAc following MFB stimulation that we observed with FSCV. Increased extracellular dopamine could result from slower dopamine uptake, but no differences in accumbal dopamine clearance were observed with FSCV by either modeling of uptake kinetics or by administration of GBR 12909, a selective DAT blocker. This suggests that the increase in extracellular dopamine in the NAc is the result of increased release. However, $U b e 3 a^{m-/ p^{+}}$mice were also less sensitive to the reward-potentiating effects of the DAT-selective reuptake blocker GBR 12909 on BSR. While this suggests that loss of UBE3A may affect dopamine receptor signaling in the NAc, the attenuated response could not be accounted for by differences in D1 or D2 receptor activity, since antagonism of both receptors elevated reward thresholds equally in both genotypes.

In contrast to that in the NAc, electrically stimulated extracellular dopamine was significantly reduced in the dorsal striatum of Ube $3 a^{m-/ p^{+}}$mice. As in that in the NAc, we observed no differences in striatal dopamine clearance with FSCV by either modeling of uptake kinetics or by administration of GBR 12909, indicating normal DAT function. This suggests that extracellular levels of dopamine are lower in dorsal striatum as a result of reduced release. One of the primary local regulators of dopamine release is negative feedback through D2 autoreceptors on dopaminergic terminals. Although D2 antagonism did not differentially affect BSR threshold in Ube $3 a^{m-/ p^{+}}$mice, the inhibitory potencies of both the D2/D3 antagonist raclopride and the D2-selective antagonist L741,626 in reducing maximum operant response rate were significantly lower, suggesting decreased D2 receptor function. Mice lacking D2 receptors exhibit some similarities to $U b e 3 a^{m-/ p^{+}}$mice, including decreased cocaine-stimulated locomotion $(23,24)$ and increased extracellular dopamine in the NAc following MFB stimulation (25), the latter effect due to decreased D2 autoreceptor function (26). However, in contrast to Ube $3 a^{m-/ p^{+}}$ mice, D2-deficient mice are also less sensitive to BSR alone (27), suggesting that the increased sensitivity to BSR and the changes in dopamine release in the NAc and dorsal striatum that we observed in Ube $3 a^{m-/ p^{+}}$ mice may be due to alterations in other signaling pathways not yet investigated.

Interestingly, some of the changes that we observed in Ube $3 \mathrm{a}^{m-/ p^{+}}$mice resemble those found in mouse models of human muta-

\section{Table 2}

Biochemical markers of dopamine

\begin{tabular}{lcccc} 
& \multicolumn{2}{c}{ NAc } & \multicolumn{2}{c}{ Dorsal striatum } \\
& WT & Ube $3 a^{m-/ p+}$ & WT & Ube $3 a^{m-/ p+}$ \\
TH protein $(\% \mathrm{WT})$ & $100 \pm 10.99$ & $116.8 \pm 13.89$ & $100 \pm 6.16$ & $102.9 \pm 11.10$ \\
& $(n=8)$ & $(n=8)$ & $(n=8)$ & $(n=8)$ \\
Dopamine $(\mathrm{nmol} / \mathrm{g})$ & $23.72 \pm 2.49$ & $25.13 \pm 2.52$ & $46.93 \pm 5.60$ & $40.93 \pm 4.63$ \\
DOPAC $(\mathrm{nmol} / \mathrm{g})$ & $1.98 \pm 0.51$ & $2.46 \pm 0.55$ & $3.37 \pm 0.93$ & $3.15 \pm 0.64$ \\
Dopamine/DOPAC & $0.091 \pm 0.03$ & $0.100 \pm 0.02$ & $0.071 \pm 0.02$ & $0.073 \pm 0.01$ \\
& $(n=5)$ & $(n=5)$ & $(n=5)$ & $(n=5)$ \\
\hline
\end{tabular}

No significant genotype differences were found (mean \pm SEM). ligase parkin (Park2) exhibit increased striatal dopamine release while also showing decreases in both spontaneous and drug-stimulated locomotion without changes in dopaminergic cell number, striatal $\mathrm{TH}$, or striatal dopamine content $(32,33)$. In contrast to loss of UBE3A, in which dopamine reuptake appears to be functionally normal, loss of either PINK1 or parkin is associated with decreased striatal DAT levels and function, while mice deficient in DJ-1 have normal DAT levels but increased dopamine reuptake and decreased behavioral and physiological sensitivity to D2 receptor agonists (30). Of note, while radionuclide imaging has confirmed loss of presynaptic dopamine in the striatum of patients with PINK1- (34), DJ-1- (35), and parkin-associated early-onset Parkinson's disease (36), no similar imaging findings or clinical measures of monoamines and their metabolites in cerebrospinal fluid have been published in studies of patients with AS.

Converging lines of evidence suggest that defects of the ubiquitin-proteasome pathway may underlie some forms of human movement disorders, particularly those involving neurodegenerative processes (37). For example, some early-onset forms of Parkinson's disease are caused by loss-of-function mutations in genes encoding parkin, PINK1, and DJ-1, while mutations in the gene encoding $\alpha$-synuclein (SNCA) have been found in both autosomal dominant and sporadic forms of the disease. As mentioned, parkin itself is an E3 ubiquitin ligase, which together with PINK1 and DJ-1 forms a complex to promote ubiquitination and degradation of parkin substrates (38). Parkin has been shown to specifically ubiquitinate and increase proteasomal turnover of a number of proteins, including O-glycosylated $\alpha$-synuclein ( $\alpha$ Sp22; ref. 39), which when overexpressed are toxic to monoaminergic neurons (40-44). Misfolded $\alpha$-synuclein or excessive levels of $\alpha$-synuclein are associated with dopaminergic cell death in Parkinson's disease and can inhibit ubiquitin-proteasomal activity $(45,46)$. Data from cell culture suggest that $\alpha$-synuclein may be a substrate for $\mathrm{UBE} 3 \mathrm{~A}$, but there is no evidence for elevated $\alpha$-synuclein levels in the absence of UBE3A in vivo (47), consistent with normal 

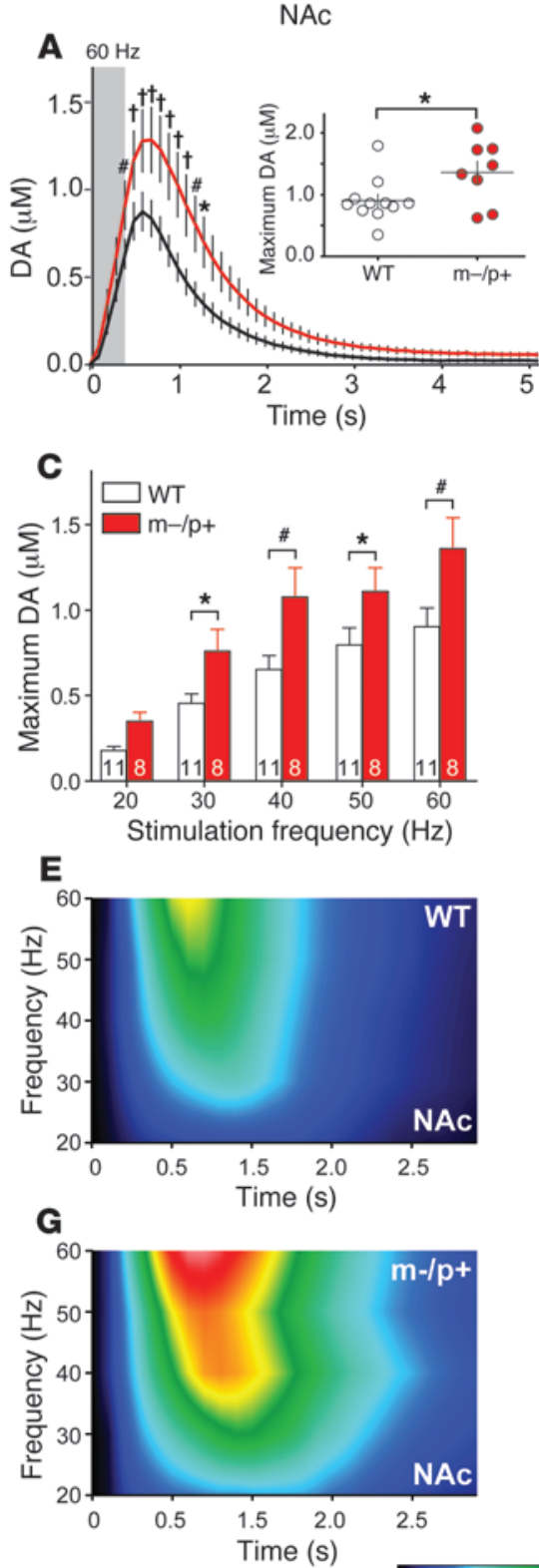

\section{Figure 5}

Dopamine release is enhanced in NAc and reduced in dorsal striatum of $U b e 3 a^{m-/ p+}$ mice. Comparison of dopamine (DA) release measured with in vivo FSCV in the (A) NAc (on left throughout) and (B) dorsal striatum (on right throughout) of Ube $3 a^{m-/ p+}$ mice and their WT littermates. (A and B) Traces represent changes in dopamine concentration evoked by $60-\mathrm{Hz}$ stimulation (stimulus duration shaded gray). Insets show peak concentration of evoked dopamine in individual animals. (C and D) Average of peak concentrations evoked by stimulation frequencies, ranging from 20 to $60 \mathrm{~Hz}$ (NAc, $30 \mathrm{~Hz}$, $P=0.05 ; 40 \mathrm{~Hz}, P=0.008 ; 50 \mathrm{~Hz}, P=0.045 ; 60$ $\mathrm{Hz}, P=0.004$; striatum, $30 \mathrm{~Hz}, P=0.006 ; 40 \mathrm{~Hz}$, $P=0.002 ; 50 \mathrm{~Hz}, P=0.015) .(\mathrm{E}-\mathrm{H})$ Extracellular dopamine concentrations following 5 discrete frequency stimulation steps, ranging from 20 to $60 \mathrm{~Hz}$ in the (E and $\mathbf{G})$ NAc or ( $\mathbf{F}$ and $\mathbf{H})$ dorsal striatum. (I and J) Response of electrically stimulated (60 $\mathrm{Hz}$ ) dopamine release to GBR $12909(20 \mathrm{mg} / \mathrm{kg})$, 30 minutes after i.p. injection. Insets show the relative increase in dopamine release (area under the curve) following GBR 12909. Error bars indicate SEM. Numbers in bars represent numbers of mice. ${ }^{*} P<0.05 ;{ }^{\#} P<0.01 ;{ }^{\dagger} P<0.001$.

numbers of midbrain dopaminergic neurons in Ube $3 a^{m-/ p^{+}}$mice. Increasing $\alpha$-synuclein expression reduces dopamine release by interfering with vesicle recycling before neurodegeneration occurs, consistent with the reduced dorsal striatal dopamine release we observed in $U b e 3 a^{m-/ p^{+}}$ mice (48). It has been shown that dopaminergic VTA neurons are resistant to the toxic effects of $\alpha$-synuclein overexpression (49), but it is unknown whether or how $\alpha$-synuclein affects dopamine release in the NAc. In light of these observations, our data further support the possibility that disorders of ubiquitination can lead to functional changes in neural circuits in the absence of neurodegeneration $(50,51)$.

L-DOPA is a mainstay of therapy for Parkinson's disease but can be accompanied by significant adverse effects. For example, patients with early-onset Parkinson's disease with parkin mutations are exquisitely sensitive to L-DOPA but are also significantly more likely to develop limiting dyskinesias and psychiatric complications while on L-DOPA therapy (52). In patients with Parkinsonism, replacement therapy with L-DOPA or directly acting dopaminergic agonists is aimed at ameliorating motor symptoms mediated by the dorsal striatum. However, such therapy consistently leads to adverse effects on reward-mediated behaviors involving the NAc (53). These observations, together with our findings that dopaminergic dysfunction in AS may be pathway specific, raise a cautionary note for ongoing clinical trials of L-DOPA in 
individuals with AS. Such studies may benefit from additional outcome measures, particularly neuropsychiatric indices, to ensure that L-DOPA treatment does not lead to unanticipated outcomes in patients with AS.

\section{Methods}

Animals. Male C57BL6/J WT and maternally deficient Ube $3 a^{m-/ p^{+}}$mice were used in all instances. These mice were generated from pairings of paternally deficient females with WT males. Age of mice ranged from P90 to P120. Mice used for ICSS were implanted at P65.7 \pm 7.8 days and fully trained for the start of behavioral assessment with cocaine at P105.5 \pm 18.1 days; raclopride at $\mathrm{P} 131.3 \pm 17.9$ days; $\mathrm{L} 741,626$ at $\mathrm{P} 155.3 \pm 16.7$ days; $\mathrm{SCH}$ 23390 at P164.5 \pm 16.2 days; or GBR 12909 at P197.3 \pm 12.7 days. Animals were kept on a 12-hour-dark/light cycle and given ad libitum access to food and water. All experiments (with the exception of Western blots) were performed blinded to genotype.

ICSS. ICSS was performed as detailed previously (54). Briefly, mice (26 WT and 27 Ube $3 a^{m-/ p^{+}}$mice) were anesthetized $(120 \mathrm{mg} / \mathrm{kg}$ ketamine, $9 \mathrm{mg} / \mathrm{kg}$ xylazine; Hospira), and $0.25 \%$ bupivacaine (Hospira) was applied to the scalp incision site. A stainless steel monopolar stimulating electrode (0.28-mm diameter; Plastics One) was stereotaxically implanted into the $\mathrm{MFB}$ at the following coordinates relative to bregma: anterior/posterior (A/P) -1.3 mm; medial/lateral (M/L) $1.1 \mathrm{~mm}$; dorsal/ventral (D/V) from skull surface $-5.0 \mathrm{~mm}(55)$. Stimulating electrodes were insulated with polyamide, leaving approximately $0.25 \mathrm{~mm}$ of the tip exposed. The electrical ground was an uninsulated stainless steel wire $(0.125-\mathrm{mm}$ diameter) wrapped around a stainless steel screw threaded into the skull. Electrode assemblies were secured to the skull with dental cement.

Following 1 week of recovery, mice were trained on a continuous (FR-1) schedule of reinforcement by the delivery of rewarding electrical stimulation (BSR) in a sound-attenuated operant chamber (Med Associates) equipped with a wheel manipulandum. Each quarter turn of the wheel was reinforced by $500 \mathrm{~ms}$ of unipolar cathodal square-wave current paired with illumination of the house light as a secondary reinforcer. Mice were presented 15 descending stimulation frequencies in discrete 0.05 inverse log steps, ranging from 126 to $25 \mathrm{~Hz}(100-\mu$ s pulse width). Each frequency trial lasted 1 minute and consisted of a 5 -second priming phase of noncontingent stimulation; 50-second active phase, during which BSR was available; and ended with a 5 -second time out, during which further responses received no additional stimulation. The minimum current intensity required to maintain responding (minimum of 40 responses per minute) for the highest 3 to 5 frequencies was determined for each individual mouse and kept constant for the duration of the experiment. For SCH 23390, raclopride, and L741,626 experiments, the current was adjusted to maintain responding for the top 8-12 frequencies. Each test session consisted of 45 minutes ( 15 frequencies for 3 repetitions) before injection and 60 minutes after injection. For GBR 12909 and L741,626, mice were placed into their home cage for 30 minutes following injection, followed by either 90 minutes ( 6 series repetitions for GBR 12909) or 75 minutes ( 5 series repetitions for L741,626) of access to BSR. BSR thresholds before injection (50\% of maximum asymptotic response rate [EF50]) were calculated from the average of the second and third series (the first series was considered a "warm-up" and was discarded) and used as daily baselines for comparison to BSR thresholds after injection.

Changes in BSR threshold were evaluated following i.p. injection of cocaine $(1.0,3.0,5.6,10.0,17.0 \mathrm{mg} / \mathrm{kg}$, calculated as the free base; $26 \mathrm{WT}$ and 27 Ube $3 a^{m-1 p^{+}}$mice; Sigma-Aldrich), raclopride (0.03, 0.1, 0.178, 0.3 $\mathrm{mg} / \mathrm{kg} ; 9 \mathrm{WT}$ and 12 Ube3 $a^{m-/ p^{+}}$mice; Sigma-Aldrich), L741,626 (1.0, 1.78, 3.0, $5.6 \mathrm{mg} / \mathrm{kg}$; 6 WT and 9 Ube3 $a^{m-p^{+}}$mice; Sigma-Aldrich); SCH 23390 (0.003, 0.01, 0.03, $0.1 \mathrm{mg} / \mathrm{kg} ; 8 \mathrm{WT}$ and 12 Ube $3 \mathrm{a}^{\mathrm{m}-\mathrm{pp}^{+}}$mice; Sigma-Aldrich);
GBR 12909 (1.0, 3.0, 10.0, $17.0 \mathrm{mg} / \mathrm{kg} ; 7 \mathrm{WT}$ and $9 \mathrm{Ube} 3 \mathrm{a}^{m-/ \mathrm{p}^{+}}$mice; Sigma-Aldrich); and normal saline or distilled water (vehicle for 741,626 ). All mice were assessed with cocaine and then divided into 2 groups, with one group of mice being tested with raclopride and L741,626 and the other group being tested with SCH 23390 and GBR 12900. Each dose was replicated 1-2 times and averaged for each mouse's behavioral response.

Locomotor behavior. Locomotion was measured in unlit sound-attenuated chambers $(27.3 \mathrm{~cm} \times 27.3 \mathrm{~cm}$; Med Associates) and quantified by beam breaks of a $16 \times 16$ photobeam infrared array. Mice were placed in the chamber for 15 minutes of habituation, injected i.p. with normal saline or cocaine, and locomotion after injection was monitored for 45 minutes. Animals were habituated to the chamber and injection stress (saline) for 4 days, and the fourth day was used as baseline locomotor activity. Following baseline determination, mice were injected with cocaine daily, and cocainestimulated locomotor activity was assessed for 6 consecutive days. Following 6 days of cocaine abstinence, during which animals remained in their home cages, locomotion after cocaine challenge was evaluated on day 7. $36 \mathrm{WT}$ and $36 \mathrm{Ube} \mathrm{a}^{m-/ p^{+}}$mice were divided into 3 separate groups of 12 , receiving either sensitizing doses of $5.6,10.0$, or $17.0 \mathrm{mg} / \mathrm{kg}$ cocaine for the entirety of the experiment.

TH immunohistochemistry. Mice (6 WT and $5 \mathrm{Ube} 3 \mathrm{a}^{m-/ p^{+}}$mice) were deeply anesthetized $(120 \mathrm{mg} / \mathrm{kg}$ pentobarbital, i.p.) and perfused transcardially with $0.1 \mathrm{M}$ PBS (pH 7.4), followed by $4 \%$ paraformaldehyde in $0.1 \mathrm{M}$ PBS. All brains were post-fixed by submersion in the same fixative for 24 hours and then cryoprotected in $10 \%$ sucrose for 24 hours followed by $30 \%$ sucrose for 24 hours. Brains were sectioned ( $40 \mu \mathrm{m}$, coronal) on a sliding microtome and stored in cryoprotectant (1.0\% polyvinylpyrrolidone $[\mathrm{w} / \mathrm{v}], 30 \%$ sucrose $[\mathrm{w} / \mathrm{v}]$, and $30 \%$ ethylene glycol $[\mathrm{v} / \mathrm{v}]$ in $0.1 \mathrm{M} \mathrm{PBS})$. Endogenous peroxidase activity was quenched with $1 \%$ hydrogen peroxide in $0.1 \mathrm{M}$ PBS for 30 minutes at room temperature. Sections were blocked in 5\% normal goat serum (NGS) and 2\% Triton X-100 in $0.1 \mathrm{M}$ PBS for 24 hours at room temperature and then incubated in rabbit $\alpha$-rat TH primary antibody (1:2,000; AB152, Millipore) in 2\% NGS and 2\% Triton for 4 days at $4{ }^{\circ} \mathrm{C}$. Sections were washed ( 3 times) in $0.1 \mathrm{M}$ PBS and incubated with HRP-conjugated goat $\alpha$-rabbit secondary antibody (1:250; 31460, Thermo Scientific) in 2\% NGS and 2\% Triton at room temperature for 3 hours. Reaction product was visualized with a DAB Peroxidase Substrate Kit (SK4100; Vector Laboratories). Sections were mounted on potassium dichromate/gelatin-subbed slides and counterstained with cresyl violet for Nissl.

Stereological quantification of cell numbers. The total number of TH-positive cells in the SNc and VTA was estimated with design-based stereology using StereoInvestigator v9.14 (MBF Bioscience) on a Microphot FXA microscope (Nikon). Every other section (section interval $=2$ ) was immunostained and counted, yielding on average 12 midbrain sections per animal. The borders of the SNc and the VTA at all rostrocaudal levels in the midbrain were delineated at low (x4) magnification based on the standard mouse atlas (55). For purposes of this study, the VTA and SNc were divided by a line connecting the medial border of the medial lemniscus, dorsally, to the medial edge of the corticospinal tract, ventrally, in all sections. Counting frames $(50 \mu \mathrm{m}$ $\times 50 \mu \mathrm{m}$ ) were randomly placed and systematically moved through a sampling grid $(120 \mu \mathrm{m} \times 120 \mu \mathrm{m})$ by the software via a motorized stage (Ludl Electronic Products). An optical disector height of $12.0 \mu \mathrm{m}$, flanked by 2.0- $\mu \mathrm{m}$ guard zones on top and bottom, was applied to all counting frames based on average section shrinkage estimates. Bright-field counting under direct visualization was performed at $\times 100$ oil magnification ( $\mathrm{NA}=1.4$ ). Estimates of the total numbers of TH-immunostained neurons were calculated using the optical fractionator method (56). For all estimates, coefficients of error $\leq 0.10$ were accepted (57). Using this protocol, results were obtained that are in agreement with estimates of numbers of dopaminergic SNc neurons in C57BL6/J mice using other methods (58). 
TH and Ube3a immunofluorescence colocalization. $2 \mathrm{WT}$ and 2 Ube $3 a^{m-/ p^{+}}$mice were transcardially perfused with $4 \%$ paraformaldehyde, and their brains were post-fixed, cryoprotected, and sectioned on a sliding microtome as described above. Tissue sections were rinsed thoroughly in Tris-buffered saline plus $0.3 \%$ Triton X-100 (TBST, pH 7.5) prior to being incubated at $80^{\circ} \mathrm{C}$ for 20 minutes in $10 \mathrm{mM}$ sodium citrate buffer ( $\mathrm{pH}$ 6.0) for antigen retrieval. Subsequently, sections were rinsed in TBST and blocked for 25 minutes in TBST containing $4 \%$ nonfat dry milk. Blocked sections were then incubated for 2 days at $4{ }^{\circ} \mathrm{C}$ in the same blocking solution containing sheep anti-TH (1:1,000; Pel-Freez) and mouse anti-Ube3a (1:200; clone 330, Sigma-Aldrich) primary antibodies. Sections were then rinsed with fresh blocking solution prior to incubation for 1 hour at room temperature in biotinylated donkey anti-mouse IgG (1:250; Jackson ImmunoResearch Laboratories Inc.) and Alexa Fluor 488 donkey anti-sheep secondary antibodies (Invitrogen). Following several rinses in TBST, sections were incubated for 1 hour at room temperature in Alexa Fluor 568 -streptavidin $(2 \mu \mathrm{g} / \mathrm{ml}$ in TBST). Tissue was mounted on subbed slides and coverslipped with DPX. Images were taken using confocal microscopy (LSM710, Zeiss).

Tissue punches. Mice were deeply anesthetized with sodium pentobarbital, and brains were snap frozen in methylbutane on dry ice. Brains were serially sectioned on a cryostat, until reaching the rostral pole of the NAc. Tissue punches (pooled from both hemispheres) were taken from the NAc and dorsolateral striatum (17 and 15 gauge, respectively). Punches were stored at $-80^{\circ} \mathrm{C}$ until use.

Western blotting. Tissue punches from the NAc and striatum $(8 \mathrm{WT}$ and

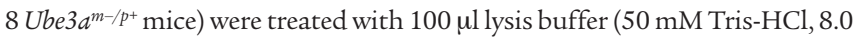
$\mathrm{pH}, 150 \mathrm{mM} \mathrm{NaCl}, 1 \%$ Triton $\mathrm{X}-100,0.1 \% \mathrm{SDS}, 0.5 \%$ sodium deoxycholate). To assess TH protein levels, $20 \mu \mathrm{g}$ of total protein lysates from WT and Ube $3 a^{m-p^{+}}$tissue homogenate were separated by $4 \%-12 \%$ SDS-polyacrylamide gel electrophoresis. Proteins were then transferred to nitrocellulose membranes, and immunoblotting was performed using a sheep anti-TH antibody (1:1,000; Pel-Freez), followed by IRDye800CW-conjugated donkey anti-sheep IgG (1:1,000; Rockland) and mouse anti- $\beta$-actin antibody (1:1,000; Sigma-Aldrich), followed by Alexa Fluor 680-conjugated goat anti-mouse IgG (1:5,000; Invitrogen). Protein bands were visualized by an Odyssey system (LI-COR Biosciences). To control for protein loading, TH protein levels were normalized to $\beta$-actin levels detected in each sample.

HPLC. Tissue punches from the NAc and dorsal striatum ( 5 WT and 6 Ube $3 a^{m-/ p^{+}}$mice) were weighed and mixed with $200 \mu \mathrm{l} 0.1 \mathrm{~N} \mathrm{HClO}_{4}$ containing $1 \mu \mathrm{M}$ hydroquinone, the internal standard. Tissue was homogenized using a sonic dismembrator (model 60; Fisher Scientific). The homogenate was then centrifuged at $10,000 \mathrm{~g}$ for 10 minutes, and the supernatant was removed and filtered using a $0.2-\mu \mathrm{m}$ syringe filter (Millex-LG; Millipore). HPLC was employed, using a modified method of Mefford et al. (59). Briefly, $20 \mu \mathrm{l}$ injections were made onto a revered-phase column $(5 \mu \mathrm{m}$, $4.6 \mathrm{~mm} \times 5 \mathrm{~mm}$; Waters Atlantis). The mobile phase contained $0.1 \mathrm{M}$ citric acid, $1 \mathrm{mM}$ sodium hexylsulfate, $0.1 \mathrm{mM}$ EDTA ( $\mathrm{pH}=3$ ), and $10 \%$ methanol as the organic modifier at a flow rate of $1.0 \mathrm{ml} / \mathrm{min}$. Neurotransmitters were detected with a thin-layer radial electrochemical cell (BASi) at a potential of $+800 \mathrm{mV}$ vs. the $\mathrm{Ag} / \mathrm{AgCl}$ reference electrode. Data were collected at $60 \mathrm{~Hz}$ using a LabVIEW strip chart recorder program (Jorgenson Lab; University of North Carolina at Chapel Hill) and homebuilt electronics. The peak area of the analyte was ratioed to the peak area of the internal standard, and the analyte concentration in the tissue was calculated. Stock solutions were made to be $10 \mathrm{mM}$ in $0.1 \mathrm{~N} \mathrm{HClO}_{4}$, and response ratios were determined daily using $2 \mu \mathrm{M}$ solution mixtures.

FSCV. Mice (NAc, $11 \mathrm{WT}$ and 8 Ube $3 a^{m-/ p^{+}}$mice; dorsal striatum, 7 WT and 9 Ube $3 a^{m-/ p^{+}}$mice) were anesthetized with urethane $(1.5 \mathrm{~g} / \mathrm{kg}$ body) and affixed in a stereotaxic frame (David Kopf Instruments). Stereotaxic craniotomies were performed, and a carbon-fiber microelectrode was implanted in either the $\mathrm{NAc}$ (from bregma, $\mathrm{A} / \mathrm{P}+1.1, \mathrm{M} / \mathrm{L}+1.2, \mathrm{D} / \mathrm{V}$ from skull surface -3.5 to -4.0 ) or dorsal striatum (from bregma, $\mathrm{A} / \mathrm{P}+0.5, \mathrm{M} / \mathrm{L}+2.5, \mathrm{D} / \mathrm{V}$ from skull surface -2.5 to -3.0$)(55)$. A bipolar stainless steel stimulating electrode, insulated to the tip (0.2-mm diameter; Plastics One), was implanted into the MFB (bregma, $\mathrm{A} / \mathrm{P}-1.2, \mathrm{M} / \mathrm{L}+1.0, \mathrm{D} / \mathrm{V}$ from skull surface -5.0 ), and an $\mathrm{Ag} / \mathrm{AgCl}$ reference electrode was implanted into the contralateral hemisphere. Computer-generated biphasic pulse trains, $2 \mathrm{~ms}$ in width and $325 \mu \mathrm{A}$ each phase, were applied through constant current stimulators (NL 800A; Neurolog, Medical Systems Corp.), for 24 pulses at 20, 30, 40, 50, and $60 \mathrm{~Hz}$ to evoke dopamine release. Stimulation-evoked release was recorded during and after the stimulation. GBR 12909 (20 mg/mg, Sigma-Aldrich) was dissolved in saline and injected i.p. at a volume of $1 \mathrm{ml} / \mathrm{kg}$.

Glass-encased, cylindrical carbon-fiber microelectrodes and $\mathrm{Ag} / \mathrm{AgCl}$ reference electrodes were prepared as described previously (60). Briefly, carbon-fiber microelectrodes were constructed by vacuum aspiration of a single T-650 carbon fiber (Thornel, Amoco Co.) into a glass capillary of 0.6-mm external diameter and 0.4-mm internal diameter (A-M Systems, Inc.). A micropipette puller (Narishige) was used to taper the glass and form a carbon-glass seal. The exposed carbon fiber was cut to approximately 100 $\mu \mathrm{m}$ in length and was soaked for 30 minutes in isopropyl alcohol to clean the surface. Application of the voltage waveform and data collection for FSCV were computer controlled and have been described in detail previously (61). Briefly, a triangular scan $(-0.4$ to $+1.3 \mathrm{~V}, 400 \mathrm{~V} / \mathrm{s})$ was repeated every $100 \mathrm{~ms}$. Data were digitized and stored on a computer using software written in LABVIEW (National Instruments). Background-subtracted cyclic voltammograms were obtained by digitally subtracting voltammograms collected during baseline recording from those collected during stimulation. A custom-built UEI potentiostat (Department of Chemistry Electronics Facility, University of North Carolina at Chapel Hill) was used. All potentials are reported compared with the $\mathrm{Ag} / \mathrm{AgCl}$ reference electrode. Signal processing (background subtraction, signal averaging, and digital filtering) (4-pole Bessel Filter, $2 \mathrm{kHz}$ ) was also performed by the software. All signals were verified as dopaminergic by the shape of their cyclic voltammogram as well as by their response to GBR 12909, a selective inhibitor of the DAT. Data were analyzed and plotted using TH-1 software.

ICSS and FSCV electrode placement verification. Mice used for ICSS were deeply anesthetized with sodium pentobarbital and intracardially perfused with $0.9 \%$ saline followed by $4 \%$ paraformaldehyde in $0.1 \mathrm{M}$ PBS. Mice used for FSCV were anesthetized with sodium pentobarbital, and brains were removed and drop fixed in $4 \%$ paraformaldehyde in $0.1 \mathrm{M}$ PBS. The brains were sectioned $(50 \mu \mathrm{m})$ on a sliding microtome, stained with cresyl violet for Nissl, and viewed under low-powered $(\times 4)$ light microscopy to determine the location of the most ventral electrode tip placements (Supplemental Figures 5 and 6).

Statistics. SigmaPlot (Systat Software) was used for statistical analysis. Mann-Whitney tests were run to compare baseline reward thresholds (EF50), baseline maximum response rates, or basal locomotor activity between genotypes. All other comparisons were based on a repeated-measures model that included a genotype-by-dose (ICSS), genotype-by-day (locomotor activity), genotype-by-second (FSCV), or genotype-by-stimulation frequency (FSCV) interaction. All genotype contrasts (Holm-Sidak) were performed based on these models and reported when significant. An additional comparison between day 1 and challenge was performed for locomotor activity experiments to determine whether sensitization occurred. Corrections for multiple comparisons were made for FSCV experiments based on the genotype-by-second model. Level of significance for all reported effects was placed at $P<0.05$.

Study approval. All experimental animal procedures were carried out according to the NIH Guide for the Care and Use of Laboratory Animals and the Society for Neuroscience Policy on the Use of Animals in Neuroscience Research 
and were approved by the Institutional Animal Care and Use Committee at the University of North Carolina at Chapel Hill.

\section{Acknowledgments}

The authors wish to thank Matthew Judson, Jason Sosa-Pagán, Megan McGuigan, and Kirk McNaughton for technical assistance with processing brains for electrode placements and immunohistochemistry. This work was supported by funding from the National Institute on Drug Abuse (DA 010900) to R.M. Wightman, from the National Institute on Alcohol Abuse and Alcoholism to C.W. Hodge (AA 014983 and AA 011605) and C.J. Malanga (AA 018335), and from the National Institute on Mental Health (MH 093372) to B.D. Philpot and by grants from the
Simons Foundation and the Angelman Syndrome Foundation to B.D. Philpot and a grant from Autism Speaks to C.J. Malanga.

Received for publication November 14, 2011, and accepted in revised form September 10, 2012.

Address correspondence to: C.J. Malanga, University of North Carolina, CB 7025, 170 Manning Drive, Chapel Hill, North Carolina 27599-7025, USA. Phone: 919.966.1683; Fax: 919.843.4576; E-mail: malangacj@neurology.unc.edu. Or to: Benjamin D. Philpot, University of North Carolina, CB 7545, 115 Mason Farm Road, Chapel Hill, North Carolina 27599-7545, USA. Phone: 919.966.0025; Fax: 919.966.3870; E-mail: bphilpot@med.unc.edu.
1. Steffenburg S, Gillberg CL, Steffenburg U, Kyllerman M. Autism in Angelman syndrome: a population-based study. Pediatr Neurol. 1996;14(2):131-136.

2. Peters SU, Beaudet AL, Madduri N, Bacino CA Autism in Angelman syndrome: implications for autism research. Clin Genet. 2004;66(6):530-536.

3. Williams CA, et al. Angelman syndrome 2005: updated consensus for diagnostic criteria. Am JMed Genet A. 2006;140(5):413-418.

4. Kishino T, Lalande M, Wagstaff J. UBE3A/E6-AP mutations cause Angelman syndrome. Nat Genet. 1997;15(1):70-73.

5. Mabb AM, Judson MC, Zylka MJ, Philpot BD. Angelman syndrome: insights into genomic imprinting and neurodevelopmental phenotypes. Trends Neurosci. 2011;34(6):293-303.

6. Knoll JH, Nicholls RD, Magenis RE, Graham JM Jr, Lalande M, Latt SA. Angelman and Prader-Willi syndromes share a common chromosome 15 deletion but differ in parental origin of the deletion. Am J Med Genet. 1989;32(2):285-290.

7. Williams CA, Zori RT, Stone JW, Gray BA, Cantu ES, Ostrer H. Maternal origin of 15q11-13 deletions in Angelman syndrome suggests a role for genomic imprinting. Am J Med Genet. 1990;35(3):350-353.

8. Rougeulle C, Glatt H, Lalande M. The Angelman syndrome candidate gene, UBE3A/E6-AP, is imprinted in brain. Nat Genet. 1997;17(1):14-15.

9. $\mathrm{Vu} \mathrm{TH}$, Hoffman AR. Imprinting of the Angelman syndrome gene, UBE3A, is restricted to brain. Nat Genet. 1997;17(1):12-13.

10. Sutcliffe JS, et al. The E6-AP ubiquitin-protein ligase (UBE3A) gene is localized within a narrowed angelman syndrome critical region. Genome Research. 1997;7(4):368-377.

11. Pelc K, Boyd SG, Cheron G, Dan B. Epilepsy in Angelman syndrome. Seizure. 2008;17(3):211-217.

12. Harbord M. Levodopa responsive Parkinsonism in adults with Angelman Syndrome. J Clin Neurosci. 2001;8(5):421-422.

13. Tan W-H. A Trial of Levodopa in Angelman Syndrome. NIH Web site. http://clinicaltrials.gov/ct2/ show/NCT01281475. Updated March 16, 2012. Accessed October 1, 2012.

14. Mulherkar SA, Jana NR. Loss of dopaminergic neurons and resulting behavioural deficits in mouse model of Angelman syndrome. Neurobiol Dis. 2010; 40(3):586-592.

15. Ferdousy F, et al. Drosophila Ube3a regulates monoamine synthesis by increasing GTP cyclohydrolase I activity via a non-ubiquitin ligase mechanism. Neurobiol Dis. 2011;41(3):669-677.

16. Philpot BD, Thompson CE, Franco L, Williams CA. Angelman syndrome: advancing the research frontier of neurodevelopmental disorders. J Neurodev Disord. 2011;3(1):50-56.

17. Cooper BR, Breese GR. A role for dopamine in the psychopharmacology of electrical self-stimulation. Natl Inst Drug Abuse Res Monogr Ser. 1975;(3):63-70.

18. Wise RA. Brain reward circuitry: insights from unsensed incentives. Neuron. 2002;36(2):229-240.

19. Benuck M, Lajtha A, Reith ME. Pharmacokinetics of systemically administered cocaine and locomotor stimulation in mice. J Pharmacol Exp Ther. 1987; 243(1):144-149.

20. Allensworth M, Saha A, Reiter LT, Heck DH. Normal social seeking behavior, hypoactivity and reduced exploratory range in a mouse model of Angelman syndrome. BMC Genet. 2011;12:7.

21. Wise RA, Rompre PP. Brain dopamine and reward. Annu Rev Psychol. 1989;40:191-225.

22. Walz NC, Baranek GT. Sensory processing patterns in persons with Angelman syndrome. Am J Occup Ther. 2006;60(4):472-479.

23. Chausmer AL, Elmer GI, Rubinstein M, Low MJ, Grandy DK, Katz JL. Cocaine-induced locomotor activity and cocaine discrimination in dopamine D2 receptor mutant mice. Psychopharmacology (Berl). 2002;163(1):54-61.

24. Welter M, Vallone D, Samad TA, Meziane H, Usiello A, Borrelli E. Absence of dopamine D2 receptors unmasks an inhibitory control over the brain circuitries activated by cocaine. Proc Natl Acad Sci U S A. 2007;104(16):6840-6845.

25. Rouge-Pont F, Usiello A, Benoit-Marand M, Gonon F, Piazza PV, Borrelli E. Changes in extracellular dopamine induced by morphine and cocaine: crucial control by D2 receptors. J Neurosci. 2002; 22(8):3293-3301.

26. Bello EP, et al. Cocaine supersensitivity and enhanced motivation for reward in mice lacking dopamine D2 autoreceptors. Nat Neurosci. 2011; 14(8):1033-1038.

27. Elmer GI, et al. Brain stimulation and morphine reward deficits in dopamine D2 receptor-deficient mice. Psychopharmacology (Berl). 2005;182(1):33-44.

28. Kitada T, et al. Impaired dopamine release and synaptic plasticity in the striatum of PINK1-deficient mice. Proc Natl Acad Sci U S A. 2007;104(27):11441-11446.

29. Lee JY, et al. Human DJ-1 and its homologs are novel glyoxalases. Hum Mol Genet. 2012;21(14):3215-3225.

30. Goldberg MS, et al. Nigrostriatal dopaminergic deficits and hypokinesia caused by inactivation of the familial Parkinsonism-linked gene DJ-1. Neuron. 2005;45(4):489-496.

31. Gispert $\mathrm{S}$, et al. Parkinson phenotype in aged PINK1-deficient mice is accompanied by progressive mitochondrial dysfunction in absence of neurodegeneration. PLoS One. 2009;4(6):e5777.

32. Itier JM, et al. Parkin gene inactivation alters behaviour and dopamine neurotransmission in the mouse. Hum Mol Genet. 2003;12(18):2277-2291.

33. Goldberg MS, et al. Parkin-deficient mice exhibit nigrostriatal deficits but not loss of dopaminergic neurons. J Biol Chem. 2003;278(44):43628-43635.

34. Khan NL, et al. Clinical and subclinical dopaminergic dysfunction in PARK6-linked parkinsonism: an ${ }^{18}$ F-dopa PET study. Ann Neurol. 2002;52(6):849-853.

35. Dekker M, et al. Clinical features and neuroimaging of PARK7-linked parkinsonism. Mov Disord.
2003;18(7):751-757

36. Scherfler C, et al. Striatal and cortical pre- and postsynaptic dopaminergic dysfunction in sporadic parkin-linked parkinsonism. Brain. 2004; 127(pt 6):1332-1342.

37. Ross CA, Pickart CM. The ubiquitin-proteasome pathway in Parkinson's disease and other neurodegenerative diseases. Trends Cell Biol. 2004; 14(12):703-711.

38. Xiong $\mathrm{H}$, et al. Parkin, PINK1, and DJ-1 form a ubiquitin E3 ligase complex promoting unfolded protein degradation. J Clin Invest. 2009;119(3):650-660.

39. Shimura $H$, et al. Ubiquitination of a new form of alpha-synuclein by parkin from human brain: implications for Parkinson's disease. Science. 2001; 293(5528):263-269.

40. Zhang Y, Gao J, Chung KK, Huang H, Dawson VL, Dawson TM. Parkin functions as an E2-dependent ubiquitin- protein ligase and promotes the degradation of the synaptic vesicle-associated protein, CDCrel-1. Proc Natl Acad Sci U S A. 2000; 97(24):13354-13359.

41. Choi P, et al. SEPT5_v2 is a parkin-binding protein. Brain Res Mol Brain Res. 2003;117(2):179-189.

42. Wang HQ, et al. Pael-R transgenic mice crossed with parkin deficient mice displayed progressive and selective catecholaminergic neuronal loss. JNeurochem. 2008;107(1):171-185.

43. Imai Y, Soda M, Inoue H, Hattori N, Mizuno Y, Takahashi R. An unfolded putative transmembrane polypeptide, which can lead to endoplasmic reticulum stress, is a substrate of Parkin. Cell. 2001; 105(7):891-902.

44. Ko HS, et al. Accumulation of the authentic parkin substrate aminoacyl-tRNA synthetase cofactor, p38/JTV-1, leads to catecholaminergic cell death. J Neurosci. 2005;25(35):7968-7978.

45. Snyder H, Mensah K, Theisler C, Lee J, Matouschek A, Wolozin B. Aggregated and monomeric alpha-synuclein bind to the S6' proteasomal protein and inhibit proteasomal function. J Biol Chem. 2003;278(14):11753-11759.

46. Tanaka Y, et al. Inducible expression of mutant alpha-synuclein decreases proteasome activity and increases sensitivity to mitochondria-dependent apoptosis. Hum Mol Genet. 2001;10(9):919-926.

47. Mulherkar SA, Sharma J, Jana NR. The ubiquitin ligase E6-AP promotes degradation of alpha-synuclein. J Neurochem. 2009;110(6):1955-1964.

48. Nemani VM, et al. Increased expression of alphasynuclein reduces neurotransmitter release by inhibiting synaptic vesicle reclustering after endocytosis. Neuron. 2010;65(1):66-79.

49. Maingay M, Romero-Ramos M, Carta M, Kirik D. Ventral tegmental area dopamine neurons are resistant to human mutant alpha-synuclein overexpression. Neurobiol Dis. 2006;23(3):522-532.

50. Shenoy SK, Lefkowitz RJ. Multifaceted roles of beta-arrestins in the regulation of seven-membrane-spanning receptor trafficking and signalling. 
Biochem J. 2003;375(pt 3):503-515.

51. Hislop JN, von Zastrow M. Role of ubiquitination in endocytic trafficking of G-protein-coupled receptors. Traffic. 2011;12(2):137-148.

52. Khan NL, et al. Parkin disease: a phenotypic study of a large case series. Brain. 2003;126(pt 6):1279-1292.

53. Macdonald PA, Monchi O. Differential effects of dopaminergic therapies on dorsal and ventral striatum in Parkinson's disease: implications for cognitive function. Parkinsons Dis. 2011;2011:572743.

54. Fish EW, Riday TT, McGuigan MM, Faccidomo S, Hodge CW, Malanga CJ. Alcohol, cocaine, and brain stimulation-reward in C57B16/J and DBA2/J mice. Alcohol Clin Exp Res. 2010;34(1):81-89.
55. Paxinos G, Franklin KBJ. The Mouse Brain In Ste reotaxic Coordinates. 2nd ed. San Diego, California, USA: Academic Press; 2001.

56. West MJ. Stereological methods for estimating the total number of neurons and synapses: issues of precision and bias. Trends Neurosci. 1999;22(2):51-61.

57. Gundersen HJ, Jensen EB. The efficiency of systematic sampling in stereology and its prediction. J Microsc. 1987;147(pt 3):229-263.

58. Baquet ZC, Williams D, Brody J, Smeyne RJ. A comparison of model-based (2D) and designbased (3D) stereological methods for estimating cell number in the substantia nigra pars compacta (SNpc) of the C57BL/6J mouse. Neuroscience. 2009;
161(4):1082-1090.

59. Mefford IN. Application of high performance liquid chromatography with electrochemical detection to neurochemical analysis: measurement of catecholamines, serotonin and metabolites in rat brain. J Neurosci Methods. 1981;3(3):207-224.

60. Cahill PS, Walker QD, Finnegan JM, Mickelson GE, Travis ER, Wightman RM. Microelectrodes for the measurement of catecholamines in biological systems. Anal Chem. 1996;68(18):3180-3186.

61. Heien ML, Phillips PE, Stuber GD, Seipel AT, Wightman RM. Overoxidation of carbon-fiber microelectrodes enhances dopamine adsorption and increases sensitivity. Analyst. 2003;128(12):1413-1419. 